\title{
Cutting Path Optimization using Tabu Search
}

\author{
Reginald Dewil, a, Pieter Vansteenwegen ${ }^{2}$ and Dirk Cattrysse ${ }^{1}$ \\ ${ }^{1}$ K.U. Leuven, Centre for Industrial Management, Traffic and Infrastructure, Belgium \\ ${ }^{2}$ Ghent University, Department of Industrial Management, Belgium \\ ${ }^{a}$ reginald.dewil@cib.kuleuven.be
}

\begin{abstract}
Keywords: Cutting Path; Tabu Search
Abstract. This paper deals with generating paths for cutting irregular parts nested on a metal sheet. The objective is to minimize the total non-cutting time for the cutter head starting at a known location, cutting all the required elements and returning to the known location. In contrast to most literature on this topic, a part does not have to be cut at once. If this reduces the total non-cutting time, it is possible to cut a number of elements on a given part, then cut other parts and then return later to finish cutting the given part. The problem is modeled as a generalized traveling salesperson problem with special precedence constraints. An initial feasible solution is generated and improved by local moves embedded in a tabu search framework. The proposed algorithm shows promising results in comparison with a commercial software package on a limited set of test cases.
\end{abstract}

\section{Introduction}

Sheet metal has practical applications in both structural components and durable consumer goods. Several sheet metal operations can be identified such as laser cutting, punching, deep drawing, bending, incremental forming, etc. Integrated production scheduling methods such as described by B. Verlinden et al. [1] aim to minimize the total costs incurred for the entire production chain. One of the outcomes of such scheduling methods is the assignment of a set of parts to a metal sheet. Automated CAM software can then create efficient nestings of these parts on the metal sheet to minimize waste material and it can provide tool paths to guide the cutter head in cutting all the nested parts. The focus of this paper is on the tool path generation. In sheet metal laser cutting, a typical cutting process can take between several minutes to several hours, depending on the number of parts on the plate, the material type, the process parameters, and the plate thickness. The time spent on actually cutting the required elements is considered independent of the chosen tool path. However the repositioning of the cutter head between cuts, called airtime motion is dependent on the chosen tool path and is to be avoided as it is non-productive time.

Earlier studies on minimizing airtime in cutting processes utilize the strategy that once the cutter head starts cutting a contour of a part, it has to be completely cut before moving to the next contour. If the cutter head is allowed to start cutting at any point on this contour, it is called a continuous cutting problem and is considered in Hoeft \& Palekar [2], Kumar [3], Dror[4], Rao et al.[5], Lee \& Kwon[6]. On the other hand, if a set of nodes is determined on beforehand, it is a generalized traveling salesperson problem with precedence constraints and is considered in $\mathrm{Han} \& \mathrm{Na}[7,8]$, Kim et al.[9] and Castelino et al. [10]. If a set of possible piercing nodes is determined beforehand and there is no obligation to completely cut a contour once started, the problem is called the endpoint cutting problem and this is the focus of this paper. Moreira et al. [11] consider a special version of this where the cutter head never stops cutting.

In the second section, the endpoint cutting problem is introduced, it is explained how it can be formulated as a generalized traveling salesperson problem with special precedence constraints and an integer program to calculate optimal solutions is presented. In the third section, two construction algorithms and three improvement heuristics are presented. In the fourth section, computational results are presented. The fifth section summarizes the concluding remarks and the last section shows some possibilities for future research. 


\section{Problem definition and formulation}

The goal of this optimization problem is to find the shortest tool path that cuts all parts from the metal sheet. However, when a contour is cut, the area within this contour detaches from the rest of the sheet and possibly shifts position. It then becomes impossible to further cut elements or parts in this detached area. This imposes precedence constraints on the tool path. A contour is considered to be cut when the last element composing that contour is cut.

The first type of precedence constraint comes from holes in parts or from the nesting phase where a part can be nested within such holes to reduce the waste area. As such it boils down to having an inner contour within an outer contour and it is required that the inner contour is cut before its outer contour is completely cut. The second type of precedence constraint originates from nesting parts with an element in common cut. Elements in common cut are a consequence of the nesting phase. By letting two contours share an element, no waste area in between the two is necessary and furthermore only one cut is necessary instead of two, reducing process time. In this case, the common cut is required to be cut, before the surrounding contour is cut. Both types of constraints are depicted in figure 1.
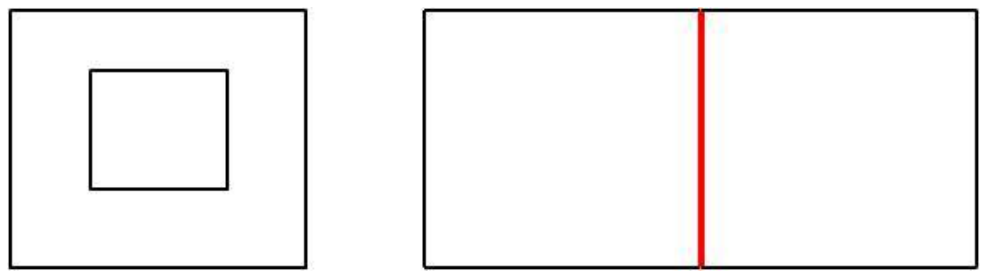

Figure 1 Precedence constraints: inner-outer contours \& lines in common cut

In a generalized traveling salesperson problem [12], a number of "cities" are grouped in districts and one tries to find the shortest path visiting exactly one city of each district. In the endpoint cutting problem, one can look at each element as a district of two "cities" where each city represents a cutting direction of the element (depicted in figure 2). The distance between a city $i$ and a city $j$ is the time required to travel from the end node of the element-direction that city $i$ is representing to the start node of the element-direction that city $\mathrm{j}$ is representing. This travel time can include the time required to decelerate and accelerate the cutter head.

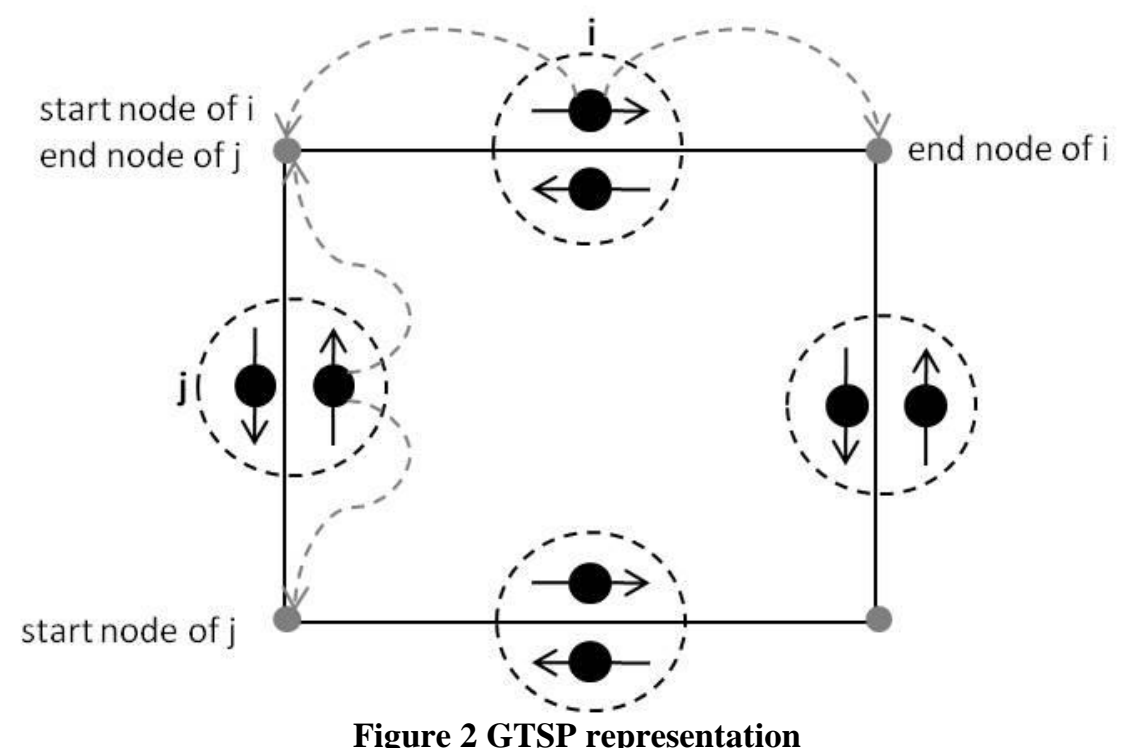

Using this representation, an integer program (IP) for the GTSP can be adjusted to incorporate the two types of precedence constraints.

$x_{i j}$ is a binary decision variable which equals one if the tool path contains a movement going from element-direction $i$ to element-direction $j$ and zero otherwise. $d_{i j}$ denotes the distance for traveling 
from element-direction $i$ to element-direction $j . y_{d e}$ is a binary variable that equals one when element $d$ precedes (not necessarily immediately) element $e$ and 0 otherwise.

$$
\begin{array}{lr}
\operatorname{Min} \sum_{i} \sum_{j} d_{i j} \times x_{i j} & \\
\sum_{i=1}^{a} x_{2 e-2, i}+x_{2 e-1, i}=1 & \forall e=2, \ldots, n \\
\sum_{i=1}^{a} x_{i, 2 e-2}+x_{i, 2 e-1}=1 & \forall e=2, \ldots, n \\
\sum_{i=1}^{a} x_{i j}=\sum_{k=1}^{a} x_{j k} & \forall j=1, \ldots, a \\
y_{d e} \geq x_{2 d-2,2 e-2}+x_{2 d-2,2 e-1}+x_{2 d-1,2 e-2}+x_{2 d-1,2 e-1} & \\
y_{d e}+y_{e d}=1 & \forall d, e=2, \ldots, n \mid d \neq e \\
y_{d e}+y_{e f}+y_{f d}+\left(x_{2 e-2,2 d-2}+x_{2 e-2,2 d-1}+x_{2 e-1,2 d-2}+x_{2 e-1,2 d-1}\right) \leq 2 & \forall d, e, f=2, \ldots, n \mid d \neq e \neq f \\
y_{1 e}=1 & \forall e=2, \ldots, n \\
y_{d e} \geq 0 & \forall d, e=2, \ldots, n \\
x_{i j} \in\{0,1\} & \forall i, j=1, \ldots, a \mid i \neq j \\
L_{I C} \times y_{I C, o} \leq \sum_{i \in I C} y_{i, o} & \forall o \in O C, \forall I C \\
y_{I C, o} \geq 0 & \forall I C, o \\
\sum_{o \in O C} y_{I C, o} \geq 1 & \forall I C \\
\sum_{o \in \text { Surroudingontour }} y_{\text {ceeo }} \geq 1 & \forall c e
\end{array}
$$

\section{IP formulation to the endpoint cutting problem}

The objective function (1) minimizes total distance traveled. Equations (2) and (3) ensure that every element is respectively exited and entered exactly once. Equation (4) states that if an elementdirection is entered, it has to be exited. Equations (5), (6), (7), (8) and (9) are sub tour elimination constraints. Equation (10) constrains the $x$ variable to a binary value. To enforce the precedence constraints, several new variables are introduced. $L_{I C}$ equals the number of elements composing contour $I C$. $y_{I C, o}$ equals one if contour $I C$ is cut before element $o$ where element $o$ is part of $I C$ 's outer contour and $y_{i, o}$ states whether element $\mathrm{i}$, which is part of the inner contour precedes element o. Equations (11), (12), and (13) ensure that an inner contour is cut before the last element of its outer contour. Equation (14) ensures that a line in common cut, is cut before at least one element of its surrounding contour.

This IP is implemented in LINDO and CPLEX and has been used to solve small instances $(<32$ elements) to optimality. However, a feasible solution wasn't found for an 84-element problem even after three days and a half of computation time. The complexity of the problem and these long calculation times lead to a heuristic solution approach.

\section{Proposed Solution Algorithm}

In this section two construction heuristics and three improvement heuristics are presented.

Furthermore, the tabu search metaheuristic is introduced, first proposed by Glover [13], which in the computational experiments guides two of the improvement heuristics. Due to confidentiality agreements with the company we are working with, no details about the algorithms can be 
discussed. In the construction algorithms the concepts of contour levels, contour ranks, and contour groups are used, depicted in figure 3.

Group 2

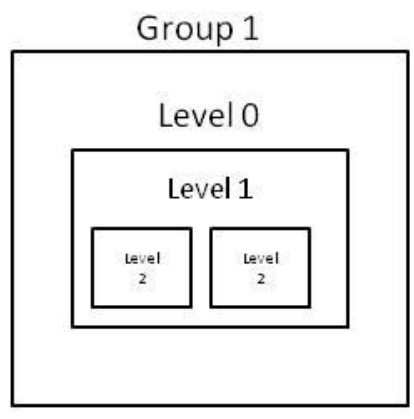

\begin{tabular}{|c|c|c|c|c|c|}
\hline $\begin{array}{c}\text { Rank } \\
0\end{array}$ & $\begin{array}{c}\text { Rank } \\
0\end{array}$ & $\begin{array}{c}\text { Rank } \\
0\end{array}$ & $\begin{array}{c}\text { Rank } \\
0\end{array}$ & $\begin{array}{c}\text { Rank } \\
0\end{array}$ & $\begin{array}{c}\text { Rank } \\
0\end{array}$ \\
\hline $\begin{array}{c}\text { Rank } \\
0\end{array}$ & $\begin{array}{c}\text { Rank } \\
1\end{array}$ & $\begin{array}{c}\text { Rank } \\
1\end{array}$ & $\begin{array}{c}\text { Rank } \\
1\end{array}$ & $\begin{array}{c}\text { Rank } \\
1\end{array}$ & $\begin{array}{c}\text { Rank } \\
0\end{array}$ \\
\hline $\begin{array}{c}\text { Rank } \\
0\end{array}$ & $\begin{array}{c}\text { Rank } \\
1\end{array}$ & $\begin{array}{c}\text { Rank } \\
2\end{array}$ & $\begin{array}{c}\text { Rank } \\
2\end{array}$ & $\begin{array}{c}\text { Rank } \\
1\end{array}$ & $\begin{array}{c}\text { Rank } \\
0\end{array}$ \\
\hline $\begin{array}{c}\text { Rank } \\
0\end{array}$ & $\begin{array}{c}\text { Rank } \\
1\end{array}$ & $\begin{array}{c}\text { Rank } \\
1\end{array}$ & $\begin{array}{c}\text { Rank } \\
1\end{array}$ & $\begin{array}{c}\text { Rank } \\
1\end{array}$ & $\begin{array}{c}\text { Rank } \\
0\end{array}$ \\
\hline $\begin{array}{c}\text { Rank } \\
0\end{array}$ & $\begin{array}{c}\text { Rank } \\
0\end{array}$ & $\begin{array}{c}\text { Rank } \\
0\end{array}$ & $\begin{array}{c}\text { Rank } \\
0\end{array}$ & $\begin{array}{c}\text { Rank } \\
0\end{array}$ & $\begin{array}{c}\text { Rank } \\
0\end{array}$ \\
\hline
\end{tabular}

Figure 3 Contour levels, ranks, and groups

Contour levels refer to the inner-outer contour relationships. A level 0 contour has no outer contour, a level 1 contour has an outer contour and a level $x$ contour has a level $x-1$ outer contour. Contour ranks come into play when there are many contours linked together with elements in common cut. Contours located deeper within such a "grid" are allocated a higher rank than those located on the outside. Contour groups are sets of contours that have an inner-outer contour relationship or share an element in common cut.

Construction Algorithms. The first construction algorithm is an insertion based algorithm (IB) (see figure 4). An insertion order is created by ordering the elements from highest to lowest (or vice versa) contour level of the contour they are part of. This is done to avoid inner-outer contour feasibility checking between contours more than one level apart. Next, a partial path going from the start node back to the start node is initialized. Then, iteratively the next element in the insertion order is taken, the feasible insertion positions in the partial path are determined, the insertion cost of each of these positions is calculated and the element is inserted in the cheapest position. Of course, for each element, the insertion costs for both cutting directions need to be calculated.

0

1

2

3

4

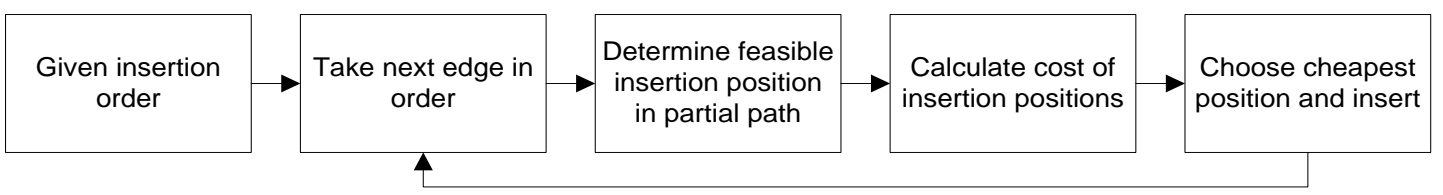

Figure 4 Construction Heuristic 1: Insertion

In the IB construction algorithm, most computation time is spent on determining the feasible insertion positions. This is because for each element, the algorithm needs to go over the contours it is part of and check whether that contour has inner or outer contours and restrict its feasible insertion positions. If the contour has an outer contour, the element needs to be inserted before the last element of that outer contour. If the contour has an inner contour, it needs to be inserted after the last element of its inner contour if the element in question is the last element of the contour. Furthermore, if there are many contours linked together through elements in common cut, many surrounding contours can be identified as depicted in figure 5. An element in common cut needs to be inserted before the last element of each of those surrounding contours. Or put otherwise, there has to be at least one element of each surrounding contour that is inserted after the element in common cut. 

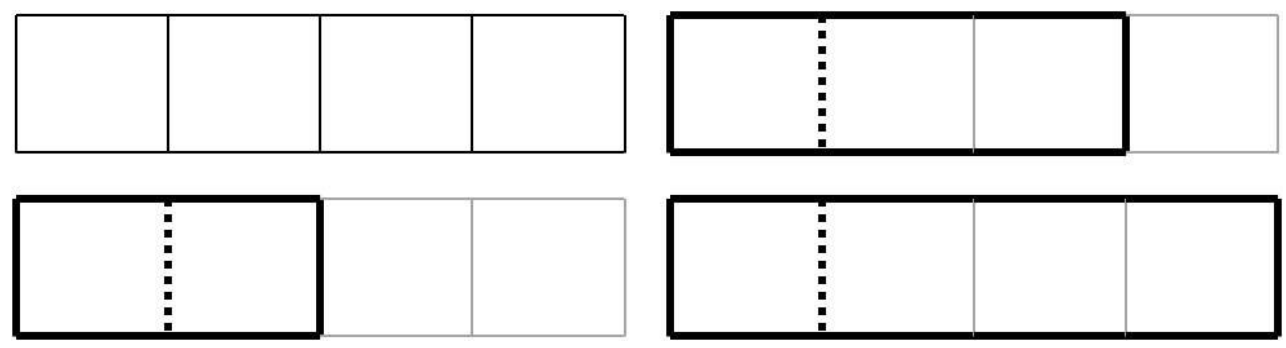

Figure 5 Surrounding Contours

To avoid the identification and feasibility checking of all these surrounding contours, a second construction heuristic was built. The second construction heuristic is called the contour order based (COB) heuristic and is depicted in figure 6. It first determines a contour order and then builds a path by sequentially adding the elements of the contours to the cutting path.

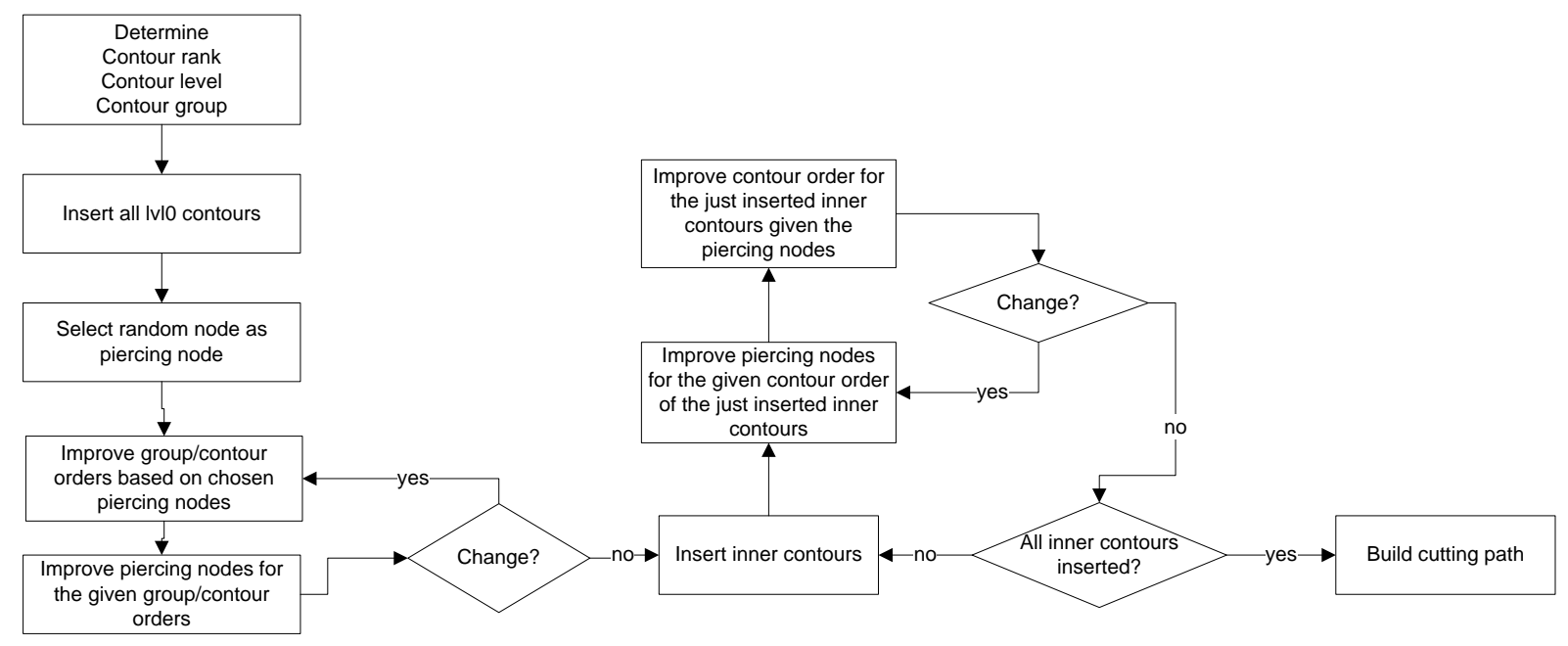

Figure 6 Contour order based construction heuristic

The algorithm works as follows:

1. All level 0 contours are placed in their respective contour groups

2. The contours are ordered within their contour groups from highest rank to lowest

3. For each contour, a random piercing node is chosen

4. The group and contour orders are improved using a greedy nearest neighbor approach using the chosen piercing nodes

5. The piercing nodes are improved, also using a greedy nearest neighbor approach, given the contour and group orders

6. If a change occurred in step 5, go back to step 4, else continue

7. Working back from the last contour in the order, inner contours are recursively added just before its respective outer contour

8. Every time all inner contours of a given outer contour are inserted, steps 3-6 are executed for this set of contours

9. If all contours are added, the path is built by sequentially adding the elements of every contour starting with the element that has the same start node as the chosen piercing node of that contour

Utilizing this construction heuristic avoids the identification and checking of surrounding contours when there are lines in common cut present, because a line in common cut is always cut before any other edge of the second contour it is part of. Consequently it is cut before its first surrounding 
contour. And if all lines in common cut are cut before their first surrounding contour, they are cut before all surrounding contours are cut.

Improvement Algorithms. The first improvement heuristic, called dir-opt, optimizes the element-directions for a given element order. This is accomplished using a dynamic programming approach suggested by Muyldermans et al. [14] for the generalized routing problem. This algorithm has a linear computational complexity.
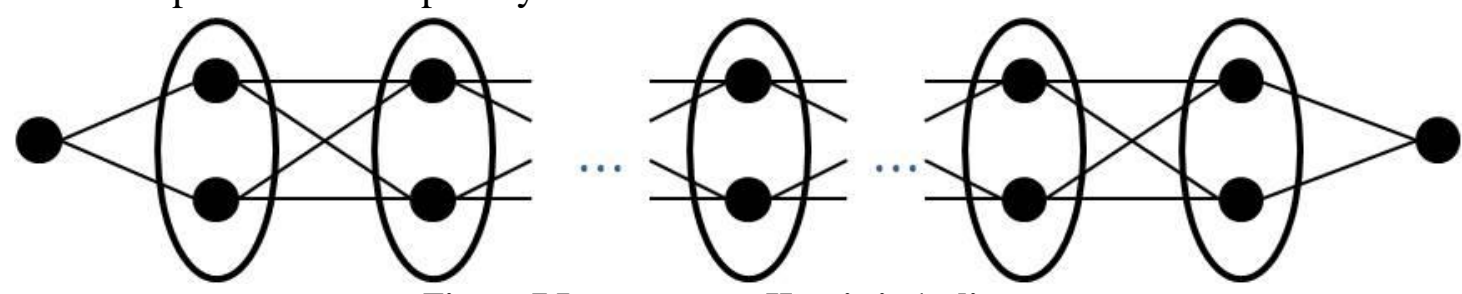

Figure 7 Improvement Heuristic 1: dir-opt

The second improvement heuristic is an Or-opt(1) move. In this move, an element is taken from the path and one of its directions is reinserted in another position (see figure 8). For the chosen element, the boundaries in between which a feasible insertion is possible are determined. For every of the feasible positions, the cost of reinserting either one of its two directions is calculated and the best insertion location is chosen.

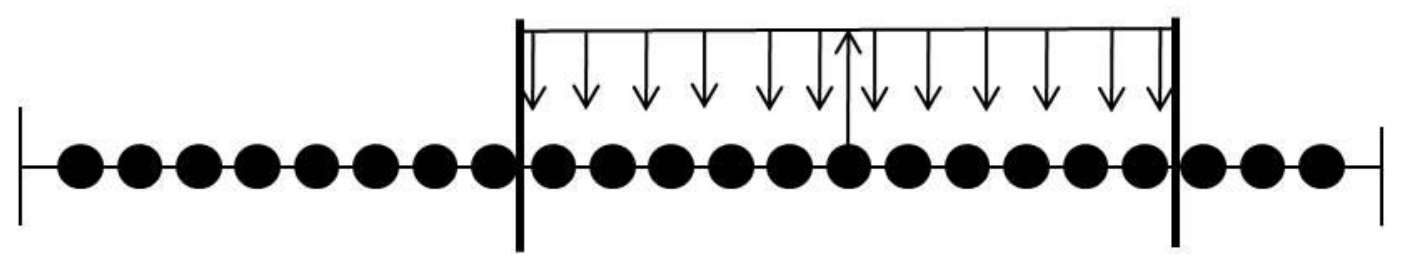

Figure 8: Improvement heuristic 2: Or-opt(1)

The third improvement heuristic is a path preserving 3-opt move (pp3opt) in which the directions of the sub paths are not reversed (figure 9). This move is proposed by Gambardella \& Dorigo [15] for solving a traveling salesperson problem with precedence constraints. In this move, a sub path is switched with an adjacent sub path.

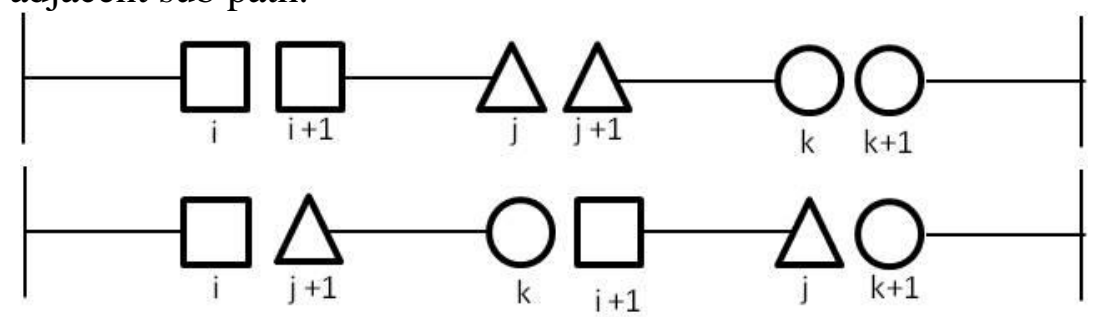

Figure 9 Improvement heuristic 3: pp3-opt

Tabu Search Framework. Both the Or-opt and pp3-opt move have a great risk of getting stuck in local optima if only improving moves are allowed. To alleviate this, a metaheuristic framework such as tabu search can be used. In every step, the costs of the possible moves in a neighborhood are calculated. If an improving move is found, this move is executed, and the reverse move is declared tabu i.e. it cannot be executed again for a number of iterations. The number of iterations during which a move is considered tabu is called the tabu tenure. However, aspiration criteria can revoke this tabu status. A typical aspiration criterion is when the move would lead to a new best solution. When a local optimum is found, thus no improving moves can be found, the least degrading non-tabu move is chosen. In this way, the incumbent solution moves away from the local optimum and may end up in a region with a different local optimum. This is repeated until a set computation time is reached. 


\section{Computational Results}

In this section the results are discussed of experiments on a set of 7 test cases depicted in figure 10. The algorithms were coded in Visual C++ and run on an Intel duo CPU 3 machine with 4GB of memory. For problems, 1, 2 and 3, tool paths using a commercial software package were also generated for comparison purposes. Problem 4 has been used in the studies of Han \& Na (1998) and Lee \& Kwon (2006) as a continuous cutting problem. Problem 5 was solved using the IP model presented in section 2 using CPLEX. Problems a280 and pr1020 are reference problems for the traveling salesperson problem which can be found on TSPlib. Inner-outer contour constraints and lines in common cut constraints are artificially added to these problems in such a way that the known optimal solution for the TSP is still feasible for the TSP with extra precedence constraints and therefore also optimal.

1
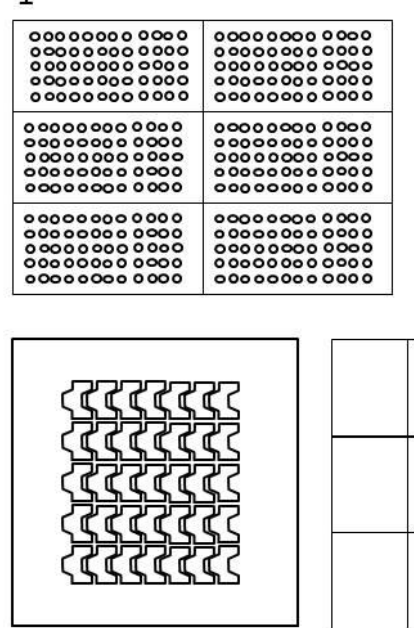

4
2

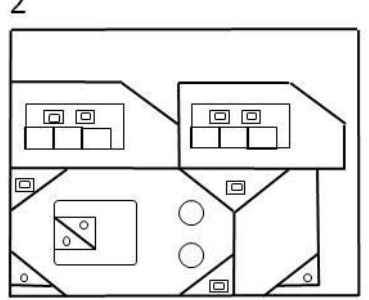

3
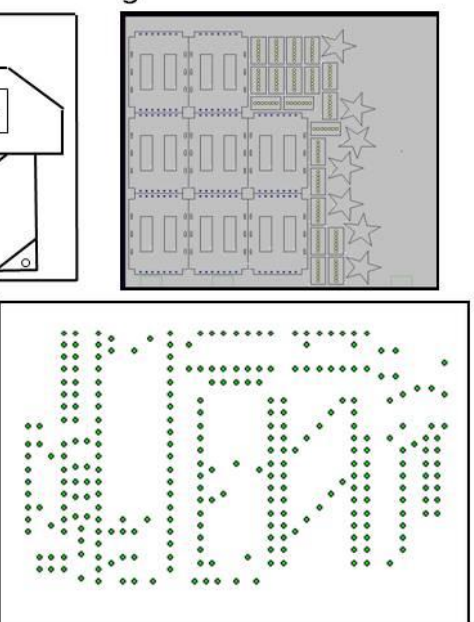

a280

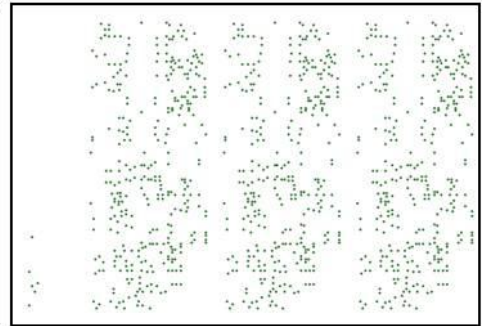

pr1020

Figure 10 Test problems

In these tests, the costs of introducing extra piercing points were disregarded as we found that relatively few extra piercing points were actually introduced in the tool paths. In the case of thin plates this has a negligible effect on the total process, while on thicker plates this can have a significant impact. Incorporating the costs of piercing points in the optimization procedure can be the focus of further research.

Since better results were obtained using dir-opt, with little increase in computation time, this improvement heuristic is always executed after running the construction heuristic. In the first series of tests, summarized in table 1.the two construction algorithms are compared for all test problems. The second column contains the number of elements present in the problem and the results shown represent only the distances of the air movements of the cutter head. 
Table 1 Results construction heuristics

\begin{tabular}{|c|c|c|c|c|c|}
\hline \multirow[b]{2}{*}{ Problem } & \multirow[b]{2}{*}{$\mathbf{n}$} & \multicolumn{2}{|c|}{ IB + dir-opt } & \multicolumn{2}{|c|}{ COB + dir-opt } \\
\hline & & Length (mm) & Time (s) & Length (mm) & (s) \\
\hline 1 & 3018 & 34265 & 1.640 & 63773 & 1.143 \\
\hline 2 & 85 & 7331 & 0.009 & 12342 & 0.001 \\
\hline 3 & 601 & 12128 & 0.062 & 29049 & 0.055 \\
\hline 4 & 421 & 384 & 0.018 & 314 & 0.015 \\
\hline 5 & 32 & 1795 & 0.023 & 2530 & 0.000 \\
\hline a280 & 280 & 3294 & 0.008 & 17168 & 0.010 \\
\hline pr1020 & 1020 & 384622 & 0.132 & 4239531 & 0.078 \\
\hline
\end{tabular}

From these tests, it can be concluded that the insertion based heuristic on average yields better quality solutions but requires a longer computation time. In the a280 and pr1020, there is a huge difference in solution quality. This is due to the fact that the contour order based heuristic cuts elements of contours one after another, but since these two problems were artificially constructed, elements of the contours are not necessarily close to one another.

In a next set of tests the initial solutions are subjected to the Or(1)-opt and pp3-opt neighborhood searches. Since a lot of time is spent on feasibility checking, a first improvement strategy for both local searches is chosen. Both neighborhood searches are tested until no further improvements could be found. The results are summarized in table 2 .

Table 2 Improvement heuristics

\begin{tabular}{|r|c|rr|rr|}
\hline & & \multicolumn{2}{|c|}{ IB + dir-opt + Or(1)opt } & \multicolumn{2}{|c|}{ IB + dir-opt + pp3opt } \\
Problem & n & Length (mm) & Time (s) & Length (mm) & Time (s) \\
\hline $\mathbf{1}$ & 3018 & 31566 & 57.373 & 30926 & 568.598 \\
$\mathbf{2}$ & 85 & 6100 & 0.016 & 7162 & 0.031 \\
$\mathbf{3}$ & 601 & 10877 & 0.616 & 10407 & 7.926 \\
$\mathbf{4}$ & 421 & 313 & 0.525 & 240 & 2.626 \\
$\mathbf{5}$ & 32 & 924 & 0.043 & 1414 & 0.044 \\
$\mathbf{a 2 8 0}$ & 280 & 3027 & 0.082 & 2875 & 0.517 \\
pr1020 & 1020 & 330349 & 11.536 & 301942 & 34.162 \\
\hline
\end{tabular}

From these tests we can conclude that both neighborhood moves are able to improve the solution quality. CPU-time increases not only as the number of elements increases, but also certain characteristics of the problem have an effect: e.g. problem 1 consists of many inner contours, which increases the number of feasibility checks before a move can be executed.

If more time is available, it can be considered to embed these local moves into a metaheuristic such as tabu search. No extensive parameter tuning was undertaken and it can be assumed that this will also improve the results. Tabu tenure was chosen as the number of elements divided by four and the metaheuristic was allowed to run for 100 seconds. Table 3 summarizes the results and a basis of comparison was chosen for each problem. For problems 1, 2 and 3, the tool path length of commercial CAD/CAM software was used as a basis for comparison. For problem 4, this was the solution found by Lee \& Kwon. . For problem 5, this was the optimal solution determined through the IP model presented in section 2. And for problems a280 and pr1020, these were the optimal solutions as presented on TSPlib. 
Table 3 Results Tabu Search

\begin{tabular}{|r|c|r|r|r|}
\hline Problem & n & Comparison & TS Or(1)opt & TS pp3opt \\
\hline $\mathbf{1}$ & 3018 & 34835 & 31389 & 32907 \\
$\mathbf{2}$ & 85 & 14629 & 4648 & 5140 \\
$\mathbf{3}$ & 601 & 21024 & 10449 & 10206 \\
$\mathbf{4}$ & 421 & 172 & 154 & 232 \\
$\mathbf{5}$ & 32 & 866 & 866 & 866 \\
$\mathbf{a 2 8 0}$ & 280 & 2579 & 2838 & 2751 \\
pr1020 & 1020 & 259045 & 320247 & 301742 \\
\hline
\end{tabular}

It can be concluded that the tabu search improves the results of the local searches. More refinement in the tabu search framework and the local search sub routines could yield further improvements. More importantly, we can conclude that considering the cutting problem as an endpoint cutting problem instead of a continuous cutting problem as in the commercial software package, considerable reductions in airtime movements can be obtained. In comparison with solutions found by the commercial software package, the tool paths found by the proposed algorithm are 3-68\% shorter. With regards to optimality, this tabu search approach is able to find the optimal solution for problems consisting of less than 32 elements and solutions within $7-24 \%$ of the known optimal for larger problems.

\section{Conclusions}

In this paper, it is shown that in contrast to most literature, it is beneficial to model the cutting problem as an endpoint cutting problem. In the endpoint cutting problem, it is allowed to cut a number of elements of a given contour, move on to cut other contours and later move back to finish cutting the given contour. An IP was formulated, but long computation times triggered a heuristic approach. Two construction and three improvement heuristics were presented. The IB construction heuristic generated shorter tool paths than the $\mathrm{COB}$ construction heuristic, but required longer computation times. The Dir-opt improvement heuristic always yielded improvements with little increase in computation time. Both the Or(1)-opt and the pp3-opt improvement heuristics were adapted to incorporate the special precedence constraints of the endpoint cutting problem and were able to improve the solution quality. The adapted neighborhood moves were embedded in a tabu search framework which was able to further improve the tool paths. Given a nesting of parts, the resulting tool paths were 3-68\% shorter than the ones found by a commercial software package. Two TSP test cases were altered into the endpoint cutting problem and the tabu search approach was able to find solutions within $7-24 \%$ of the optimal solution.

\section{Discussion and Future Research}

Although promising results were obtained with the proposed algorithm, further research to improve the algorithm and to test a larger number of problems is warranted. Furthermore, in practical cutting processes additional considerations are to be made in generating a tool path. In this paper, the costs of adding extra piercing points that are required to start cutting in a new section of the metal sheet were not considered. This cost needs to be weighed against the advantages of less air movements. However, this trade off can be complicated by the introduction of precuts (if one passes an element where one later wants to start cutting again, the cutter head can make a small detour and make a cut, to eliminate the need for a piercing point later on) or bridges (instead of lifting the cutter head and making a piercing point in an element close by, the cutter head can just cut towards the next element). The time spent on creating a piercing point, precut or bridge is dependent on the material type, cutting process and sheet thickness. The dangers of collisions were also not considered, where the cutter head can collide with a cut part jutting out of the surface of the sheet. Both of the two 
previous extensions to the cutter problem come down to the distances between any two points on the sheet to be dynamic, i.e. the cost of moving between two points is dependent on the path already cut at that moment. Another major extension, which also gets addressed in Han and $\mathrm{Na}$ [6][7] and Kim et al. [8] is heat accumulation and conduction in sheet metal laser cutting that can cause material deterioration and burn off. This especially becomes a problem in thick sheets and in sharp corners but regarding the problem as an endpoint cutting problem allows for more flexibility in avoiding high temperature areas of the plate.

\section{Acknowledgements}

The authors acknowledge the financial support from IWT-Vlaanderen (Instituut voor de Aanmoediging van Innovatie door Wetenschap en Technologie Vlaanderen).

\section{References}

[1] B. Verlinden et al.: Multiprocessor Scheduling: Theory and Applications, chapter 20. (2007)

[2] J. Hoeft and U.S. Palekar: IIE Transactions. Vol. 29 (1997), pp. 719-731

[3] D.V.S. Kumar: International Journal of Production Research. Vol. 36 (1998), pp. 1901-1916

[4] M. Dror: IIE Transactions. Vol. 31 (1999), pp. 271-274

[5] Y. Rao, G. Huang, P. Li, X. Shao, and D. Yu: The International Journal of Advanced Manufacturing Technology. Vol. 33 (2006), pp. 436-448

[6] M.-K. Lee and K.-B. Kwon: International Journal of Production Research. Vol. 44 (2006), pp. $5307-5326$

[7] G.-chan Han and S.-joo Na: Journal of Manufacturing Processes. Vol. 1 (1999), pp. 54-61

[8] G.-chan Han and S.-joo Na: Journal of Manufacturing Systems. Vol. 1 (1999), pp. 62-70

[9] Y. Kim, K. Gotoh, and M. Toyosada: Journal of Marine Science and Technology. Vol. 9 (2004), pp. 70-79

[10] K. Castelino, R. D'Souza, and P.K. Wright: Journal of Manufacturing Systems. Vol. 22 (2003), p. 173-180

[11] L. Moreira, J. Oliveira, A. Gomes, and J. Ferreira: Computers \& Operations Research. Vol. 34 (2007), pp. 3281-3294

[12] S.S. Srivastava, S. Kumar, R.C. Carg, and P. Sen: CORS. Vol. 7 (1969), pp. 97-101

[13] F. Glover and M. Laguna in: Tabu Search, Kluwer Academic Publishers, Boston (1999)

[14] L. Muyldermans, P. Beullens, D. Cattrysse, and D. Van Oudheusden: Operations Research, Vol. 53 (2005), pp. 982-995

[15] L.M. Gambardella and M. Dorigo: INFORMS Journal on Computing (2000) 